\title{
27 BURDEN OF ROAD TRAFFIC INJURIES IN EGYPT: STRENGTHS AND WEAKNESSES OF DATA SOURCES
}

doi:10.1136/injuryprev-2012-040590u.27

HF El-Sayed, P Puvanachandra, AA Hyder, R Saad, S Eldawy, N Al-Gasseer. Faculty of Medicine, Suez Canal University, Egypt

Study objective To define the health impact and risk factors of RTIs in Egypt, and to identify the strengths and weaknesses of each data source to improve the current RTI data systems.

Methods A two-pronged approach was undertaken to assess the burden of RTIs in Egypt. Thorough literature review was performed, and comprehensive exploration of data sources pertaining RTIs in Egypt was undertaken, and data were critically analysed.

Results The national RTIs fatality rate as reported by the mortality information systems in 2008 was 10.58 per 100000 population. However, inherent gaps in the data presented owing to the lack of data and limited coverage of the data systems. Many of the data sources which report fatalities due to RTIs do not follow the internationally recommended definition of fatality which includes 30 day follow-up, which results in a significant underrepresentation of deaths. Furthermore, there is non-standardisation between data sets between the health, traffic police and transport sectors. Modeled road traffic fatality rate of 41.6 deaths per 100000 population was estimated in Egypt, which is about four times the reported estimate. These data gaps also appeared in estimation of number of crashes and RTIs injuries.

Conclusions The review clearly highlight the significant burden that RTIs in Egypt. Mechanisms to improve the quality of data, the use of ICD-10 coding in injury surveillance system, enhance 
consistency across sources, generate policy-friendly reporting systems, and the conduct of special studies on costing and disability will add to the evidence base in Egypt. 